\title{
On the Cause of Indol Convulsions in Warm=blooded Animals.
}

\author{
By \\ Bin-ichi Yanai. \\ (矢丙 敏 一) \\ (From the Pharnacological Laboratory of Prof. S. Yagi, \\ Tohoku Imperial University, Sendai.)
}

According to Herter ${ }^{1)}$ and $\mathrm{Bieb} \mathbf{l}^{29}$ indol acts on warm-blooded animals leading to convulsion; however no concrete description concerning it is presented by them. The convulsions under indol may arise from an increased excitability of the motor elements of the spinal cord, just as is proposed by myself ${ }^{33}$ for explaining the occurrence of convulsions appearing in frogs after the same poison. It is also not improbable, that they may have another cause than that in the case of frogs, for pyrrol, from which indol is derived, seems after Tomi$\mathrm{naga} \mathrm{a}^{4)}$ to affect the central nervous system of warm-blooded animals in an entirely different way than in the case of cold-blooded animals. It is therefore of interest to investigate the appearance and the cause of the convulsions in warm-blooded animals under indol, especially in making out the behaviour of the pyrrol nucleus contained in the molecule of the pyrrol derivative.

\section{General Symptoms.}

The experiments were performed on mice and rabbits, and indol, dissolved in water or olive oil according to concentrations desired, was given to them subcutaneously.

1. Mouse. Soon after the administration of a large dose of indol to a mouse, it took a crouching position breathing slowly and irregu-

1) Herter, Journ. of Exp. Med., 1899, 4, 307.

2) Bie bl, Dentsch. Ztschr. f. Chir., 1929, 218, 135.

3) Y a n a i, Tohoku Journ. of Exp. Med, 1935, 25, 385.

4) To min ag a, ditto, 1933, 21, 444, 458 and 461 . 
larly, as a result of local irritation due perhaps to the injection itself. After about ten minutes, transient fascicular twitchings began to appear repeatedly here and there over the body without any order. Thereafter they increased steadily in intensity and frequency to such an extent, that the animal was no longer able to keep quiet, but was compelled to move back and forth staggering restlessly. Then the breathing became intensely frequent and shallow. Later on the twitchings commenced to be interrupted by a repeated onset of general clonic convulsions lasting for a short time. The convulsions became more and more intense, so that in an advanced stage the animal tumbled about during the attack, like an elastic ball bouncing. But sooner or later the convulsions became gradually weaker until they disappeared. The animal then lay on its side, but still showed muscular twitchings. When the twitchings had almost completely ceased, the animal was breathing very slowly and shallowly, and showed neither spontaneous nor reflex movements. Several hours after the administration the respiration ceased without resulting in agony. The lachrymatory and salivary secretions increased to a stage where the clonic convulsions appeared, but decreased with their relapse. The minimum lethal dose was found to be 3.5 to $4.0 \mathrm{mgrms}$. per $10 \mathrm{grms}$. of the body weight leading to death within twenty four hours, and the minimum dose required to produce the fascicular twitchings corresponded to about half of the former dose.

2. Rabbit. The symptoms seen in a rabbit receiving a large dose of indol were analogous in feature to those observed in the mouse under the same condition, though in particulars there were some differences between the two cases. In this case the clonic convulsions occuring paroxysmally in the course of the fascicular twitching were not so intense as in the case of the mouse. During their attack frequent blinking and nystagmus were detected. At the stage where the attack of the clonic convulsions disappeared, while the fascicular twitchings were still persisting, the animal lay on its side showing at intervals so-called swimming and galloping movements of the extremities. The pupils constricted while the twitchings were continuing, but during the attack of the clonic convulsions they dilated slightly. The minimum lethal dose was 0.8 to 1.2 grms. per $\mathrm{kgrm}$. of the weight, death occurring within twenty four hours.

In these experiments it is seen, that symptoms produced by indol in the mouse and the rabbit resemble each other, and can be classified in two independent groups, the one including exciting phenomena such 
as the fascicular twitchings, general clonic convulsions, and the other including paralytic phenomena such as weakening and ultimate failure of the spontaneous, reflex and respiratory movements. Though in respect to the symptoms consisting of the two defined groups of the phenomena, the indol poisoning in the mouse and the rabbit is the same as that in the frog and the toad, there are seemingly at least some differences in the exciting phenomena between these two groups of animals. Consequently it is not yet certain, whether the exciting. phenomena appearing in the mouse and the rabbit are of the same origin as those of the frog and the toad or not.

\section{Analysis of the Convulsions.}

1. The fascicular twitchings appearing transitorily, but frequently in the indol poisoning of the mouse and the rabbit bear some resemblance to those produced by physostigmine or nicotine, so that in the first place experiments dealt with the peripheral action of the poison.

After injection of indol, in a quantity sufficient to give rise to the general clonic convulsions, into the subcutaneous or muscular tissues in the thigh of a rabbit, the fascicular twitchings began to appear in several parts of the body almost simultaneously, without regard to the distance from the place injected. When the twitchings developed distinctly in the hind legs, the section of the sciatic nerve of one leg led to their abolition from the distal part of the same leg. In a later stage the clonic convulsions set in in other parts of the body than the distal part of the leg, which showed merely passive movements during the attack of the convulsions.

In another rabbit a muscle of one thigh was exposed and a small quantity of a 0.01 per cent indol solution was injected into the superficial layer of it. But no twitching of the muscle could be produced after the injection.

The twitchings and clonic convulsions due to indol, therefore, have no relation whatever to its peripheral action, so that attention must be turned in another direction.

2. When a mouse was attacked by fascicular twitchings and clonic convulsions after the administration of indol, the animal was fastened to a plate, and under the performance of artificial respiration the spinal cord was divided in the neck. Soon after the section the twitchings as well as convulsions disappeared completely from the 
rear part of the body, while they continued in the head, though somewhat weaker than before the section.

In a rabbit the spinal cord was divided across between the first and second dorsal vertebrae, bleeding being prevented as completely as possible. After the animal had been allowed a few hours for recovering from shock due to the operation, a sufficient quantity of indol was given to it subcutaneously. In this case, while muscular twitchings, clonic convulsions, swimming movements and other exciting phenomena set in in the front half of the body, just as in the usual indol poisoning, no such phenomena could be found in the rear half. But the latter part underwent a peculiar change in reflex phenomena, as follows: Before the poisoning, mechanical stimulation applied to the sole of the foot gave rise to such movements of the leg, to which it was attached, as would remove or avoid the stimulant object, but was able only occasionally to provoke movements of the other leg, the movements consisting merely of a feeble extension and flexion. But when the toxic symptoms in the front part of the body became manifest, the stimulation for evoking reflex movements in the leg of the same side needed to be very much stronger than that required before the poisoning. The movements thus produced being compounded of flexions and extensions were not far removed from the clonic convulsions of the leg in the usual indol poisoning. In such strength the stimulation was always effective in causing movements of the other leg, similar to those of the leg stimulated, and fascicular twitchings over the rear part of the trunk.

The peculiar phenomena seen in this experiment, that in spite of the decrease in the reflex excitability, the movements produced by stimulation of a certain part of a hind leg appear as clonic convulsions of both legs and as fascicular twitchings over the trunk, are essentially the same as those observed in the poisoning of the spinal frog, and seem to be interpreted only by assuming that the excitability of the sensory elements in the spinal cord decreases under the action of the poison, while on the contrary that of the motor increases. And the latter change is probably enough for producing the fascicular twitchings and clonic convulsions, if the motor elements are brought into action by the usual impulse from the higher centres. But considering the fact that the same symptoms appear also in areas of the body receiving innervation from the facial and oculomotor nerves, it is certain that motor centres situated higher than the spinal cord undergo similar change from the poison. In order to determine 
the seats of the centres acted on, the following experiments were carried out.

3. The cerebral hemispheres of a rabbit were exposed, indol applied in an olive oil solution to their surfaces. When solutions higher than 2.5 per cent was used distinct symptoms followed after about thirty minutes. The animal then became quiet and assumed a crouching position. It showed no movements unless the strongest stimulation was given. The pupils constricted slightly. But neither fascicular twitchings nor clonic convulsions could be detected in any area of the body within the course of the experiment.

A rabbit which had been deprived of its cerebrum twenty four hour's before, was given a large dose of indol subcutaneously. The symptoms arising from it, however, were not different from those of the animal with intact brain not only in their appearance but also in their intensity.

These experiments indicate clearly that the cerebrum also plays no important role in the occurrence of the symptoms mentioned.

4. When the brain of a rabbit was cut across in a transversal plane running just behind the corpora quadrigemina and corpora mamillaria, and the front part of it was removed, the animal lay on its side showing the decerebrated rigidity after Sherrington. In such an animal, subcutaneous injection of a large dose of indol was followed by typical fascicular twitchings and by decrease of the rigidity. At the stage, in which the rigidity conspicuously weakened, general clonic convulsions and trismus also occured at intervals, though they were not as intense as in the poisoning of the animal with intact brain. Mechanical stimulation applied to the body was then able to evoke general convulsions attended by fascicular twitchings. In a still more advanced stage where the rigidity disappeared almost completely the animal showed feeble, but perceptible swimming and galloping movemets.

Therefore it is certain, that the ganglions in the pons are affected by the poison so as to take an important part in producing the symptoms in question. The ganglion cells may in this case be directly stimulated by the poison. But considering its action on the spinal cord it is rather probable that their excitability is increased, but to such an extent that they are easily replaced in an immense excitement by even the slightest stimulus of any kind, so that the twitchings and convulsions occur apparently spontaneously.

The results of the experiments performed on mice and rabbits 
with indol may be briefly summerized as follows: The poison acts on the motor elements of the spinal cord and especially intensively on the ganglions of the pons to increase their excitability, so that any usual impulse from the higher centres lead to fascicular twitchings, clonic convulsions and other exciting phenomena. Whereas the reflex excitability is simultaneously lowered by the action of the poison decreasing the excitability of the sensory elements at least in the spinal cord.

The same may hold good with indol poisoning in the frog and the toad, but in these animals the action on the spinal cord being more prominent than that on the ganglions of the pons, the latter action may hide behind the former.

Pyrrol, contained in the molecule of indol as a nucleus, is stated by the previous worker to have an action stimulating the motor elements in the brain of the warm-blooded animal, without affecting the spinal cord. But, when the behaviour of the poison towards the frog is taken into account, it can not be accepted without some suspicion, that the spinal cord of the warm-blooded animal escapes absolutely from the action of the poison. It is highly probable that the cord is also affected by the poison, but so slightly, that it can be practically disregarded. On this supposition it may be said, that the action of indol is essential to the pyrrol nucleus contained in the molecule of it, the intensity however, being exaggerated by the benzene nucleus exsisting in it. 